\title{
Stress induced martensitic transformation in CuAIZnMn polycrystals investigated by in situ neutron diffraction
}

\author{
P. Sittner, P. Lukas ${ }^{1}$, M.R. Daymond ${ }^{2}$, V. Novak and G.M. Swallowe ${ }^{3}$ \\ Institute of Physics, Na Slovance 2, 18221 Prague 8, Czech Republic \\ ${ }^{1}$ Nuclear Physics Institute, 25068 Rez, Czech Republic \\ 2 ISIS Facility, Rutherford Appleton Laboratory, Chilton, Didcot, Oxon OX11 OQX, U.K. \\ ${ }^{3}$ Loughborough University, Loughborough, Leicestershire LE11 3TU, U.K.
}

\begin{abstract}
The results of in-situ neutron diffraction investigation of stress induced martensitic transformation in CuAlZnMn shape memory alloy polycrystal are reported. Time-of-flight diffraction spectra were measured during temporary stopovers along two successive tensile pseudoelastic cycles. The integral intensities, positions and widths of multiple individual austenite and martensite $h k l$-reflections from the recorded spectra were evaluated by single peak fits. The evaluated peak profile parameters were used to calculate the lattice strains in the austenite phase and volume fractions of the austenite phase evolving during the pseudoelastic cycles. The obtained $h k l$-lattice plane responses were related to the elastic anisotropy of the cubic austenite phase and to the transformation anisotropy of the $\beta_{1} \rightarrow \beta_{1}^{\prime}$ martensitic transformation in CuAIZnMn using a selfconsistent crystallographic model of SMA polycrystals. The mechanism of the load partition among polycrytal grains has been discussed and claimed to be a major obstacle for the stress driven transformability of the polycrystalline Cu-based SMAs It has been found that significant redistribution of stress and large intergranular stresses but no strong transformation induced texture should be expected in Cu based SMA polycrystals transforming pseudoelastically in tension.
\end{abstract}

\section{INTRODUCTION}

When shape memory alloy /SMA/ polycrystal undergoes stress induced martensitic transformation /SIMT/, the compatibility of strains and continuity of stress across grain boundaries must be fulfilled. Since SMAs are often elastically anisotropic and the SIMT is also a strongly anisotropic process [1,2], it might be in fact very difficult for some SMA polycrystals to sustain larger recoverable strains in polycrystalline state [3], even if their theoretical crystallographic transformation strains are of the order of $10 \%$. We are interested in this paper in the stress redistribution among grains of SMA polycrystal undergoing SIMT in tensile pseudoelastic cycles. Recent developments of in-situ neutron diffraction technique of strain measurement have allowed to study this experimentally as we will demonstrate in this paper.

Due to the large penetration depths of thermal neutrons, neutron diffraction is a suitable tool for nondestructive in-situ measurement of elastic strains (and evaluation of stresses) within the bulk of large polycrystal specimens. Diffraction strain measurements rely on the precise measurement of the $d_{h k l^{-}}$ spacing of particularly oriented crystal planes. Knowing the stress free lattice spacing $d_{0, h k l}$, the lattice strain $\varepsilon_{h k t}$ can be calculated according to equation (1).

$$
\varepsilon_{h k l}=\frac{d_{h k l}-d_{0, h k l}}{d_{0, h k l}}=\frac{\Delta d_{h k l}}{d_{0, h k l}}
$$

The diffraction evaluated strains are: i) elastic strains only, ii) determined selectively only from those grains of the specimen which are suitably oriented, iii) averaged strain values over those grains, and iv) generally only one component of the elastic strain tensor can be evaluated from a diffraction experiment with a single specimen orientation with respect to the scattering vector. The lattice strain $\varepsilon_{h k l}$ can be measured from analysis of individual diffraction peaks within the diffraction spectra recorded during insitu tensile deformation. Multiplying the lattice strains $\varepsilon_{h k l}$ parallel to the load axis direction by the Young 
modulus of the bulk material, an average value of the corresponding component of the macrostress tensor in the $h k l$-families of grains of the polycrystal is obtained. We will assume uniaxial tensile state of stress in the embedded polycrystal grains in this paper. However, if the material is elastically anisotropic, the relationship between the lattice strain and macroscopic stress is not that straightforward [4,5], and this is particularly true if inelastic deformation occurs. Firstly, the orientation dependence of the Young modulus, $E_{h k l}$, has to be taken into account. The single crystal moduli $E_{h k l}$, can be calculated (Eq. 2 applies for cubic materials only) from the single crystal elastic constants $C_{i j}$.

$$
\frac{1}{E_{h k l}}=\frac{C_{11}+C_{12}}{\left(C_{11}-C_{12}\right)\left(C_{11}+2 C_{12}\right)}-2\left(\frac{1}{C_{11}-C_{12}}-\frac{1}{2 C_{44}}\right) A_{h k l}, A_{h k l}=\frac{h^{2} k^{2}+h^{2} l^{2}+k^{k} l^{2}}{\left(h^{2}+k^{2}+l^{2}\right)^{2}}
$$

However in polycrystals, the redistribution of the stress among particularly oriented $h k l$-grains means that the measured lattice strains $\varepsilon_{h k l}$ still can not be easily related to the macroscopic stress. While, for plastically deforming solids, the problem of the evolution of $h k l$-dependent lattice strains with the macroscopic stress and strain (load partition) has been dealt with in many papers both theoretically $[6,7]$ and experimentally [8], for martensitically transforming solids, only pioneering experimental works of Vaidyanathan et al. [9] on TiNi alloys and our preliminary conference reports [10] on $\mathrm{CuAlZnMn}$ exist. In this paper, we will focus our attention on the mechanism of the load sharing among grains of pseudoelastically transforming SMA polycrystal. The experimental information obtained from the in situ neutron diffraction experiment will be analyzed using a selfconsistent crystallographic model of SMA polycrystals. A journal paper detailing more experimental results and

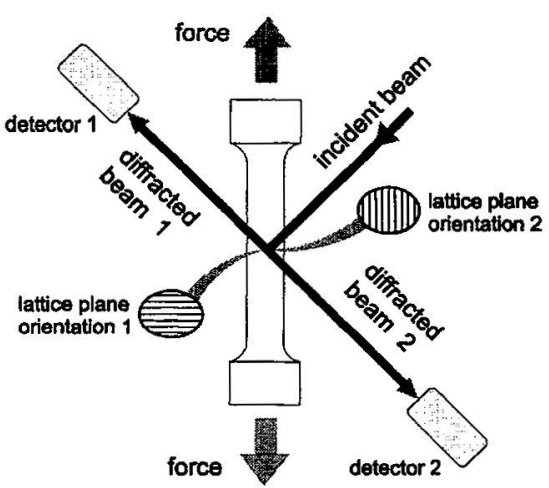

Figure 1: Arrangement of the neutron diffraction experiment. The spectra called axial and radial are collected by detectors 1 and 2 , respectively. providing further details of the diffraction experiment is currently under preparation.

\section{EXPERIMENTAL PROCEDURES}

The experiments reported in this work were performed on cylindrical bar shaped specimens (Fig.1, gauge length $=25 \mathrm{~mm}$, diameter $=8 \mathrm{~mm}$ ) made of the $\mathrm{CuAl}_{10} \mathrm{Zn}_{5} \mathrm{Mn}_{5}$ (wt.\% ) SMA polycrystal (grain size $\sim 100 \mu \mathrm{m}$ ). Transformation temperatures obtained following the heat treatment $-T=700^{\circ} \mathrm{C}$ for 2 hours and water quenching - were $M_{s}=-30^{\circ} \mathrm{C}$, $M_{f}=-58^{\circ} \mathrm{C}, A_{s}=-30^{\circ} \mathrm{C}, A_{f}=-15^{\circ} \mathrm{C}$. The CuAlZnMn alloy, as a representative of $\mathrm{Cu}$-based SMAs, undergoes stress induced $\beta_{1} \rightarrow \beta_{1}$ martensitic transformation in tension. Elastic constants of the austenite $\beta_{1}$ phase were measured by pulse-echo ultrasonic technique on $\mathrm{CuAlZnMn}$ single crystal as $C_{11}=154 \mathrm{GPa}, C_{12}=112 \mathrm{GPa}, C_{44}=77 \mathrm{GPa}$. Texture

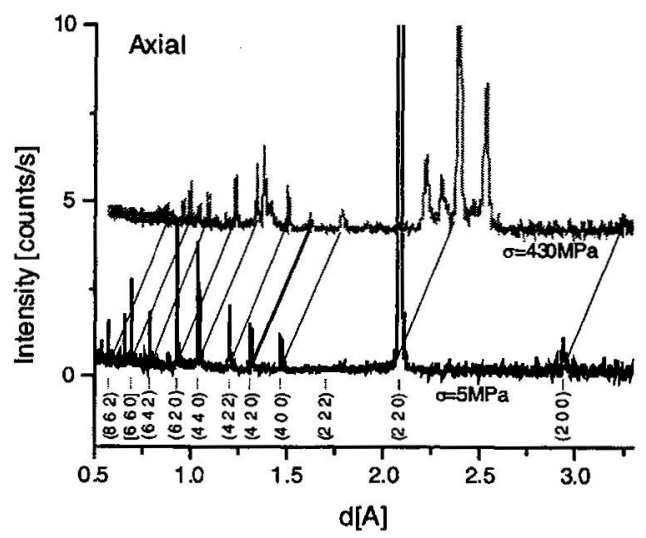

Figure 2: Neutron diffraction spectra of the CuAlZnMn polycrystalin axial configuration collected under tensile stress $\sigma_{G}=5 \mathrm{MPa}$ and $\sigma_{G}=430 \mathrm{MPa}$ at room temperature $T>A_{f}$ Only significant austenite reflections are indexed. analysis by means of neutron diffraction, performed on neutron diffractometer at NPI Rez, has shown a week [100] fiber texture aligned with the bar axis. The in-situ neutron diffraction experiment was carried out on pulsed neutron spallation source at ISIS - RAL Chilton, UK using the time-of-flight /TOF/ method on a dedicated diffractometer ENGIN. Neutron diffraction spectra were collected during temporary stopovers along two successive tensile loading cycles at 
$T=295 \mathrm{~K}$. The load was imposed by an Instron 8800 servohydraulic tester operated in the load control mode that is installed at the diffractometer. In a TOF experiment, the wide range of neutron wavelengths (velocities) in the incident beam enables the simultaneous recording of a complete diffraction spectrum consisting of many reflections (see Fig. 2). The peaks originate from families of grains oriented in such a way that the given $h k l$-lattice planes diffract to the detector in a slightly different time of flight. Geometrical arrangement of the diffraction experiment (Fig. 1) is of crucial importance for understanding the results reported in this work. The tensile loading axis was inclined $45^{\circ}$ to the incident neutron beam and two opposing $2 \Theta=90^{\circ}$ detector banks collected simultaneously the neutrons diffracted by the lattice planes of the specimen parallel and perpendicular to the loading axis (see figure 1). This allows simultaneous measurements of the $h k l$-lattice spacings with normals oriented: i) parallel (spectra denoted as radial) and ii) perpendicular (spectra denoted as axial) to the tensile load axis. Each significant austenite and martensite reflections found in the measured spectra were analyzed by single peak fits using four parameter asymmetric EMG profile function. Integral intensity, $I_{h k l}$, center of gravity of the profile peak position, $d_{h k l}$, and profile width parameter $F W H M_{h k l}$ were evaluated. The lattice strains of individual austenitic $h k l$-reflections were evaluated from the peak shifts according to equation (1). It was assumed that the annealed and quenched specimen is free of any residual stresses and the lattice spacings measured in as quenched state at zero stress were hence used as initial $d_{0, h k l}$ values. The normalized integral intensity parameter, $I_{h k l} I_{0, h k l}$ provides information on the advance of SIMT in the $h k l$-families of grains.

\section{RESULTS OF IN-SITU NEUTRON DIFFRACTION EXPERIMENT}

The points on the two tensile pseudoelastic curves of the CuAlZnMn polycrystal in figure 3 denote the stressstrain states at which the diffraction spectra (Fig. 2) were measured. Due to the space limitation, we will focus only on the analysis of axial spectra ( $h k l$-vectors parallel to the load axis). Only significant reflections of the austenite phase are indexed in figure 2. Table 1 gives a list of the 9 studied austenite reflections and parameters evaluated from the fitted diffraction profiles. Partial results of the profile fitting of axial austenite [220] reflections (Fig. 4) show the evolution of normalized integral intensity $I_{h k l} I_{0, h k l}$ and lattice strain $\varepsilon_{h k l}$ with the applied macroscopic stress, $\sigma_{G}$. The integral intensity of the [220] peak starts to decrease at $\sigma_{G} \sim 320 \mathrm{MPa}$, clearly

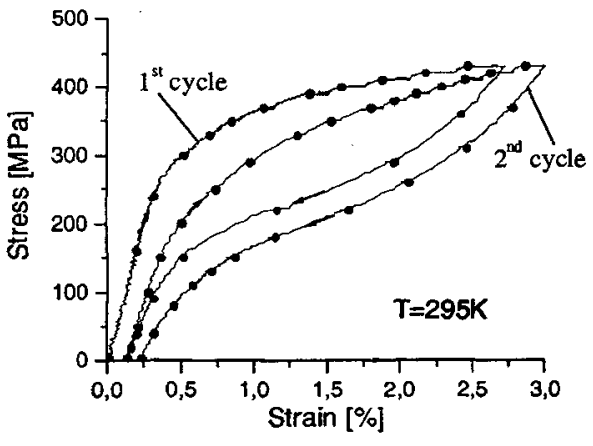

Figure 3: Two tensile pseudoelastic cycles on CuAlZnMn polycrystal at $T=295 \mathrm{~K}$ with denoted diffraction measurement points.
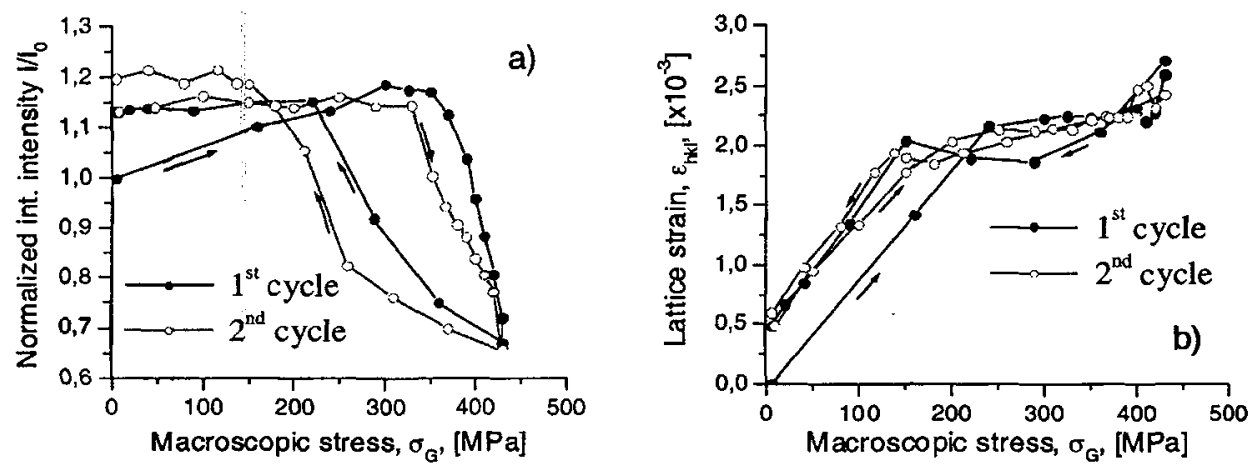

Figure 4: Lattice plane response of [220] axial reflection in the first and second pseudoelastic tensile load cycles on CuAlZnMn polycrystal: a) normalized integral intensity, b) lattice strain. Data points correspond to those denoted in Fig 3. 
due to the stress induced martensitic transformation starting to consume the austenite phase. The intensity evolves hysteretically with the stress (Fig. 4a) and linearly with the strain in the tensile load cycle. This would be expected since the peak intensity relates to the austenite phase volume fraction. The lattice strain $\varepsilon_{h k l}$, however, evolves also hysteretically (Fig $4 \mathrm{~b}$ ) with both the macroscopic stress and strain. This is a specific yet unknown SMA behavior, and, as such, it must be given further attention. The lattice strain evolution is linear in the early elastic range and but deviates later from the linearity. The onset of the deviation (Fig. 4b) clearly precedes the fall of the intensity (Fig 4a), appearance of the martensite peaks in the spectra, and most importantly, the start of the transformation flow (Fig.3).

Residual strain, $\varepsilon_{h k l}{ }^{\text {res }}=0.0005$ has not been recovered following the first tensile cycle. In the second cycle, an almost closed $\varepsilon_{h k l}-\sigma_{G}$ loop with narrow hysteresis has been observed. Notice that, even if the macroscopic stresses at which the transformation proceeds (Fig. 3) are very different in the first and second tensile cycles, the diffraction measured lattice strains (Fig. $4 \mathrm{~b}$ ) in the transformation plateau $\varepsilon_{h k l}^{\text {plat }}$ on the $\varepsilon_{h k l}-\sigma_{\mathrm{G}}$ curve are almost equal $\left(\varepsilon_{h k l} l^{\text {plat }} \sim 2 \times 10^{-3}\right)$. This can be interpreted as experimental evidence of the residual stress effect in pseudoelasticity, since the diffraction evaluated lattice strain, in fact, measures an averaged local stress, $\sigma_{h k l}=\varepsilon_{h k l} E_{h k l}=\sigma_{G+} \sigma_{h k l}^{\text {res }}$ that is due to sum of the macroscopic stress, $\sigma_{G}$, and residual stress $\sigma_{h k l}{ }^{\text {res }}=\varepsilon_{h k l}{ }^{\text {res }} E_{h k l}$, associated with the residual strain, $\varepsilon_{h k l}{ }^{\text {res }}$, left behind from the previous tensile load cycle. Since the macroscopic stress driving the SIMT in the second cycle is much lower, it may be argued that the tensile residual stresses $\sigma_{h k l}{ }^{\text {res }}$ (specific for the $h k l$-families of grains as will be shown later) assists the SIMT in the second and further tensile load cycles. Problem is that the oriented residual stresses $\sigma_{h k l}{ }^{r e s}$ in SMAs are poorly established. They likely reside within the austenite volume fraction that went through the SIMT in the first cycle. This austenite volume transforms preferentially in the second cycle, and the $\sigma_{h k l}{ }^{\text {res }}$ hence disappears with the austenite phase transforming to martensite - i.e. the effect is in fact neither "residual" (evolves with the progress of the SIMT in the polycrystal) and possibly nor the due to the stress at all (the unstable austenite lattice may remain distorted near the $T_{0}$ equilibrium temperature). Nevertheless, let us continue to use the notion "residual stress" to explain straightforwardly the decrease of the $\sigma_{G}$ in the second tensile cycle. The equality of $\varepsilon_{h k l}{ }^{\text {plat }}$ in the first and second cycles is understandable based on the well known fact that SIMT requires at particular temperature a well defined constant stress to proceed. The new experimental information is that this possibly applies also for an embedded grain of the SMA polycrystal loaded even cyclically.

Table 1: Parameters of $h k l$-lattice plane responses. $A_{h k l}$ and $E_{h k l}$ are respectively the orientation dependent anisotropy coefficient and Young modulus calculated by Eq.(2), $1 / \varepsilon^{t}{ }_{h k l}$ is a calculated measure proportional to the transformation stress $\sigma_{h k l}^{\prime r}$ in tension $\left(\varepsilon_{h k l}^{\prime}\right.$ is theoretical transformation strain due to the $\beta_{1} \rightarrow \beta_{1}^{\prime}$ transformation [11]). $I_{p, h k l} / /_{0, h k]}$ characterizes the maximum decrease of the normalized integral intensity of the $h k l$-reflection due to the SIMT. $D_{h k l}$ is

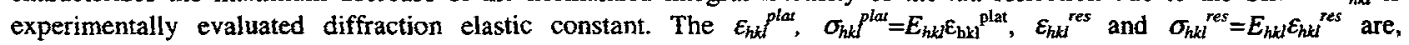
respectively, the $h k l$-lattice strain and stress at the transformation plateau and the $h k l$-residual strain and stress following the first tensile cycle. All are averaged values for the corresponding family of $h k l$-grains.

\begin{tabular}{|c|c|c|c|c|c|c|c|c|c|}
\hline \multirow{2}{*}{$\begin{array}{c}\text { Austenite } \\
\text { peak } \\
\text { [hkl] }\end{array}$} & \multicolumn{3}{|c|}{ Theory } & \multicolumn{6}{|c|}{ Experiment } \\
\hline & $A_{h k l}$ & \begin{tabular}{|l|}
$E_{h k l}$ \\
{$[\mathrm{GPa}]$} \\
\end{tabular} & $1 / \varepsilon_{h k l}^{T r}$ & $I_{p, h k l} / I_{0, h k l}$ & $\begin{array}{c}D_{h k l} \\
{[\mathrm{GPa}]}\end{array}$ & $\begin{array}{l}\varepsilon_{h k}^{\text {plat }} \\
{\left[10^{-3}\right]}\end{array}$ & $\begin{array}{l}\sigma^{\text {plat }} h k \\
{[\mathrm{MPa}]}\end{array}$ & $\begin{array}{c}\varepsilon_{h k l}{ }^{\text {res }} \\
{\left[10^{-3}\right]}\end{array}$ & $\begin{array}{c}\sigma_{h k l}{ }^{\text {res }} \\
{[\mathrm{MPa}]}\end{array}$ \\
\hline [200] & 0 & 59.7 & 12.1 & - & 64 & 3.3 & 197 & 0.83 & 50 \\
\hline$[400]$ & 0 & 59.7 & 12.1 & - & 59 & 3.25 & 194 & 0.76 & 45 \\
\hline [620] & 0.09 & 73.3 & 11.7 & 0.51 & 89.5 & 3.55 & 260 & 1.03 & 75 \\
\hline [420] & 0.16 & 89.2 & 12.3 & 0.57 & 84.4 & 2.95 & 263 & 0.87 & 77 \\
\hline [840] & 0.16 & 89.2 & 12.3 & 0.5 & 85 & 3.07 & 261 & 0.41 & 37 \\
\hline [220] & 0.25 & 123.5 & 16.2 & 0.72 & 113 & 2.26 & 279 & 0.48 & 59 \\
\hline [440] & 0.25 & 123.5 & 16.2 & 0.67 & 115.5 & 2.25 & 278 & 0.43 & 53 \\
\hline [422] & 0.25 & 123.5 & 17.9 & 0.82 & 110 & 2.68 & 331 & 0.27 & 33 \\
\hline [642] & 0.25 & 123.5 & 15.4 & 0.7 & 132.1 & 2.23 & 275 & 0.4 & 49 \\
\hline
\end{tabular}



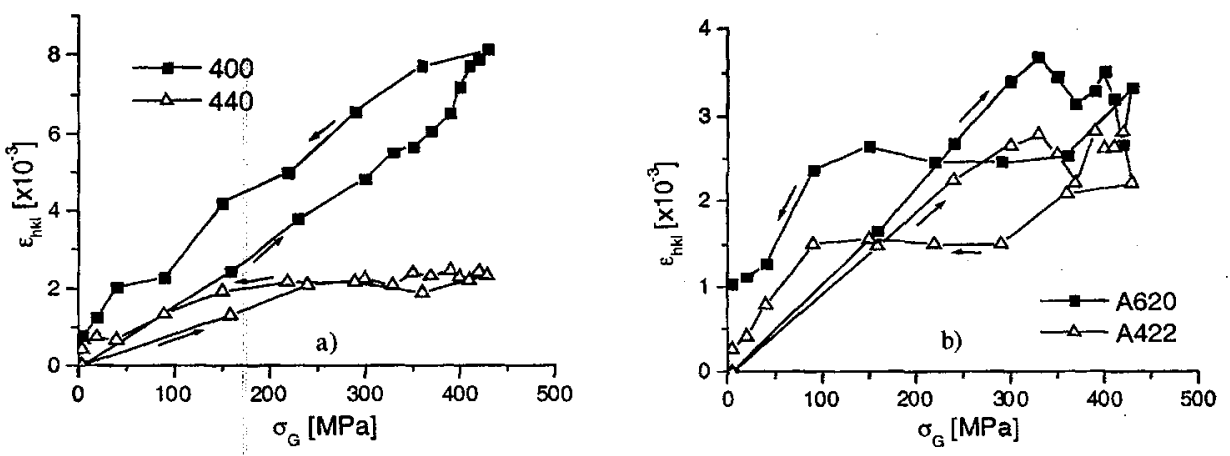

Figure 5: Lattice strain responses of a) [400],[440] and b) [620],[422] axial reflections during the first pseudoelastic cycle on CuAlZnMn polycrystal in dependence on the macroscopic stress. Data points correspond to those denoted in Fig 3.

The present diffraction experiment, however, was carried out mainly to gather a survey on the responses of multiple reflections in the CuAlZnMn spectra. This is important since results of the single reflection (as discussed above) apply only for the particular $h k l$-families of grains but not for the whole polycrystal. An example of the lattice strain responses of other four $h k l$-reflections is given in figure 5. The responses are assumed to be related to the i) elastic anisotropy, ii) transformation anisotropy and iii) load sharing between the variously oriented polycrystal grains. Most of the 9 observed axial $h k l$ responses were qualitatively somehow similar to the [220] peak behavior, except that of the [400] peak. The [100] direction of the austenite cubic lattice is the elastically softest one (Fig. 6b) and also the very soft one for the $\beta_{l} \rightarrow \beta_{l}$, transformation in Cu-based SMA (Fig. 6a, at constant temperature, the transformation stress $\sigma_{h k l}^{t r} \sim 1 / \varepsilon^{t r}{ }_{h k l}$ can be considered). The elastically hardest [664], [222] and [444] peaks in the axial spectrum were unfortunately not intensive enough for reliable strain measurements. The diffraction results given in the $\varepsilon_{h k l}-\sigma_{G}$ representation (Figs. $4 \mathrm{~b}, 5 \mathrm{~b}, \mathrm{~d}$ ) are commonly used in the neutron strain measurements [5] to determine experimentally the $h k l$-dependent diffraction elastic constants $D_{h k l}$ from the linear part of the $\varepsilon_{h k l}-\sigma_{G}$ dependence. The values of $D_{h k l}=d \varepsilon_{h k l} / d \sigma_{G}$ (Table 1) determined from the linear elastic range correspond approximately but not exactly (due to the load partition in the polycrystal) with the $E_{k k l}$ values calculated by Eq.(2). Note that the individual $h k l$-responses (Table 1, Figs 4,5) differ in: i) $\left.D_{h k l}, i i\right) \varepsilon_{h k l}^{\text {plat }}$ - lattice strain at which the deviation from linearity on the $\varepsilon_{h k l}-\sigma_{G}$ curve appears, iii) type of the $\varepsilon_{h k l}-\sigma_{G}$ non-linearity following the start of the SIMT, iv) $\varepsilon_{h k l}$ hysteresis width, v) parameter $I_{p, h k l} / I_{0, h k l}$ characterizing the maximal decrease of the normalized integral intensity due to the SIMT, and vi) $\varepsilon_{h k l}^{\text {res }}$ residual strain left behind the first tensile load cycle. Two additional parameters $\sigma_{k k l}{ }^{\text {plat }}=E_{h k l} \varepsilon_{\mathrm{hkl}}{ }^{\text {plat }}$, and $\sigma_{h k l}{ }^{r e s}=E_{h k l} \varepsilon_{h k l}{ }^{\text {res }}$ in Table 1 are the average $h k l$-component stress in the transformation plateau and $h k l$-residual stress.

Information on the load partition in polycrystal pseudoleasticity and progress of SIMT in the $h k l$ families of grains is contained within the experimental data of individual $h k l$-responses (Table 1, Figs 4,5 ). However, care has to be taken when drawing conclusions from figures 4,5 , since these relate the component values (average values for given $h k l$-families of grains) and macroscopic values $\left(\varepsilon_{G}, \sigma_{G}\right)$, and can be very easily misinterpreted. As discussed in the introduction, it is assumed that the $\varepsilon_{h k l}-\sigma_{\mathrm{G}}$ nonlinearities and hysteresis of the observed $\varepsilon_{h k l}-\sigma_{G}$ lattice responses (Fig. 4,5, Table 1) originate from the elastic anisotropy (Fig. 6b), transformation anisotropy associated with the SIMT (Fig. 6a) and with the intrinsic reversibility and hysteresis of the martensitic transformation. The problem to solve is hence to relate these anisotropies to the experimental results in Figs 4,5 and Table 1. For this purpose, we have developed a selfconsistent micromechanics model of polycrystal transformation in which the anisotropies and SIMT deformation mechanism are included. It is based on the Crystallographic model of SMA [11] published very recently. In both these models, the SMA polycrystal is assumed to be represented by an aggregate of $N$ oriented dimensionless discrete domains that deform and transform according to the 
elastic law and $h k l$-specific stress-temperature non-equilibrium diagrams calculated for each oriented domain. Individual domains are defined (have neighbours in the aggregate set up) only in the orientation space, not in the real space. Uniaxial stress state in each domain and kinematic non-hardenning hysteretic response of the domains due to the SIMT are considered (forward and reverse transformations in the domains proceed at constant local stress level by definition). There is no fitting parameters used in the model. In accord with the selfconsistent approach, the rates $d \sigma_{G} / d t$ and $d \varepsilon_{G} d t$ are equal to the macroscopic selfconsistent average of the rates of the corresponding $h k l$-domain values. The results of the model simulation of the tensile pseudoelastic test (Fig. 7) are shown for elastic constants and material parameters of CuAlZnMn given in section 2 and in Ref. [11], $T=295 \mathrm{~K}, N=13000$, and randomly oriented domains are randomly distributed in the aggregate set up.

\section{DISCUSSION}

The CuAlZnMn polycrystal loaded in tension exhibits reversible hysteretic stress-strain response with "hardening" like transformation flow (Fig. 3) and the model simulates this qualitatively very well $\left(\sigma_{G}-\varepsilon_{G}\right.$ curve in figure $7 \mathrm{a}$ ). The simulated macroscopic $\sigma_{G}$ - $\varepsilon_{G}$ responses compared with simulated $h k l$-responses in Figs. 7a give an idea about the load partition. Qualitative agreement between $\varepsilon_{h k l}-\sigma_{G}$ dependencies obtained experimentally (Figs. 4,5) and theoretically (Fig. 7b) has been also achieved. We assume that this is primarily due to the ability of the model to treat properly the load partition phenomena taking place in the transforming SMA polycrystal. In order to clarify the mechanism of the load sharing among polycrystal grain, it is very beneficial to inspect how the applied load is distributed among the oriented domains of the transforming aggregate. Three stress stages can be distinguished in which the domains behave differently: I) $0-200 \mathrm{MPa}$, II) $200-280 \mathrm{MPa}$, and III) $280-380 \mathrm{MPa}$. In the stage I, $\sigma_{G}<200 \mathrm{MPa}$, only elastic anisotropy is responsible for the stress redistribution in elastically deforming domains. In the stage II, $\sigma_{G} \sim 200-280 \mathrm{MPa}$, the SIMT starts in isolated events in some individual domains in which the transformation stress level has been reached, but does not progress continuously. The local stress in the domain, in which the transformation event has taken place, instantly falls in a response, since the load is immediately transferred to the neighboring domains and the SIMT in that domain thus temporarily stops. The $\sigma_{G}$ (prescribed in the incremental step simulation procedure) then has to raise significantly in subsequent calculation steps before the transformation stress of that domain is reached again and the transformation may there continue. The onset of the stage II is recognized as the onset of the deviations from linearity on most of the $\varepsilon_{h k l}-\sigma_{G}$ curves. In order to drive the large scale transformation of the aggregate ahead (stage III, $\sigma_{G}>280 \mathrm{MPa}$ ), the local stresses in most of domains must not fall in a response to the transformation events taking place in them. This is possible in the selfconsistent model only if majority of the domains becomes just ready to transform with their local stresses adjusted to the corresponding $h k l$ dependent transformation stresses. Before such a situation is reached, however, significant redistribution of the local stresses in individual domains has to take place in stage II. Consequently, due to the specific strong elastic and transformation anisotropy of the CuAlZnMn (Fig. 6), this means that the domains requiring transformation

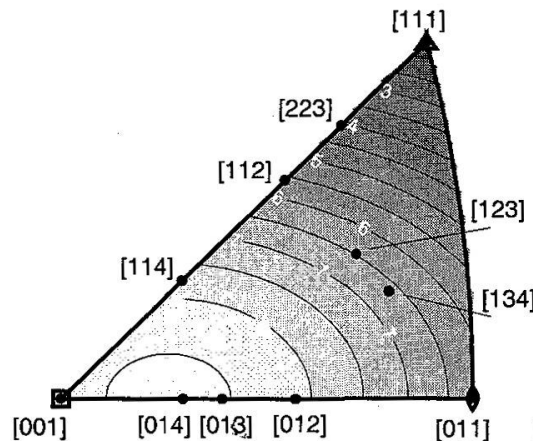

(a)

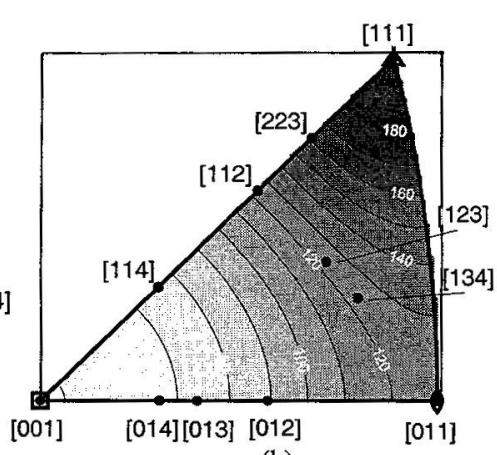

(b)

Figure 6: Orientation dependence of transformation strain, $\varepsilon_{h k l}{ }_{h k l}$ (a) and Young modulus, $E_{h k l}$, (b) in CuAlZnMn alloy calculated according to crystallography of the $\beta_{1} \rightarrow \beta_{1}^{\prime}$ SIMT [11] and equation (2), respectively. The experimentally studied $h k l$ reflections are denoted. 
(orientations near the [111] corner) are capable and are forced to share much larger part of the applied load than the other domains (compare Fig. 7a). The stress redistribution in stages II and III is thus governed not only by the elastic and transformation anisotropy, but mainly by the kinematic nonhardening hysteretic responses of the domains assumed in the selfconsistent polycrystal model.

The macroscopic stress $\sigma_{G}$ required to trigger the transformation flow of the aggregate is significantly larger in the selfconsistent model compared to the original constant stress version [11]. Since the aggregate domains are able to transform cooperatively in the large scale transformation only if the macroscopic stress steadily increases, the just outlined load partitioning mechanism leads also to the apparent macroscopic "hardening" behavior of the aggregate in the tensile test. In spite of that, however, the local stresses in the domains remain constant (oscillating around their specific transformation stress levels) while the transformation proceeds in them. Looking at the almost linear [100] lattice strain response $\varepsilon_{h k l}-\sigma_{G}$ in figures $7 \mathrm{~b}$ and $5 \mathrm{~d}$, however, one may doubt that the [100] domains (grains) indeed transform at the constant local stress level. They do (in the model by definition) but arrive at the critical transformation stress individually at very different macroscopic stresses $\sigma_{G}$. This happens in the selfconsistent model because the local stresses in the individual domains may, for some $h k l$-components, depend significantly on the location of the particular domain in the aggregate (on the load axis orientation of its neighbors). The nearly linear $\varepsilon_{h k l}-\sigma_{G}$ response hence results from the averaging of the responses of all [100] domains (grains) in the selfconsistent model (experiment), respectively. Domains oriented near the [111] comer don't transform in the early stage III at all, in spite of the very high and continuously growing local stresses in them, since these remain still below the $\sigma_{h k l}^{t r}$ stress level (Fig.6a) even when the large scale transformation already proceeds. Nevertheless, deviation from linearity appears on the $\varepsilon_{I J I}-\sigma_{G}$ early in stage II (Fig 7b) and has opposite sense than that of the [011] and most other domains.

Since the model simulations reproduced well the lattice plane responses, suppose that the above outlined mechanism of the load partition applies for the transforming CuAlZnMn polycrystal. Its pseudoelastic "hardening" (Fig 3), though apparently similar to the hardening of the common plastically deforming solids, appears to have a completely different physical origin. Instead of martensite variant interactions causing real physical hardening of the domains (frequently considered, in an analogy to the plastic deformation, in micromechanics models of SMA), the load partitioning during the cooperative transformation of the polycrystal grains is proposed here to be primarily responsible for the apparent "hardening" of the CuAlZnMn SMA polycrystal. The neutron diffraction experiment provides clear evidence of the anisotropy and stress redistribution phenomena. Compare the $D_{h k l,} I_{p, h k /} / l_{0, h k l}$ and $\sigma_{h k l} l^{p l a t}$ in Table 1. Significant stress redistribution in stage II is inevitably accompanied by the build up of intergranular stresses in the polycrystal. First deviations of the experimental $\varepsilon_{h k l}-\sigma_{\mathrm{G}}$ dependencies from linearity (Figs. 4b) appearing at stresses $\sigma_{G} \sim 240 \mathrm{MPa}$ suggest the occurrence of large intergranular
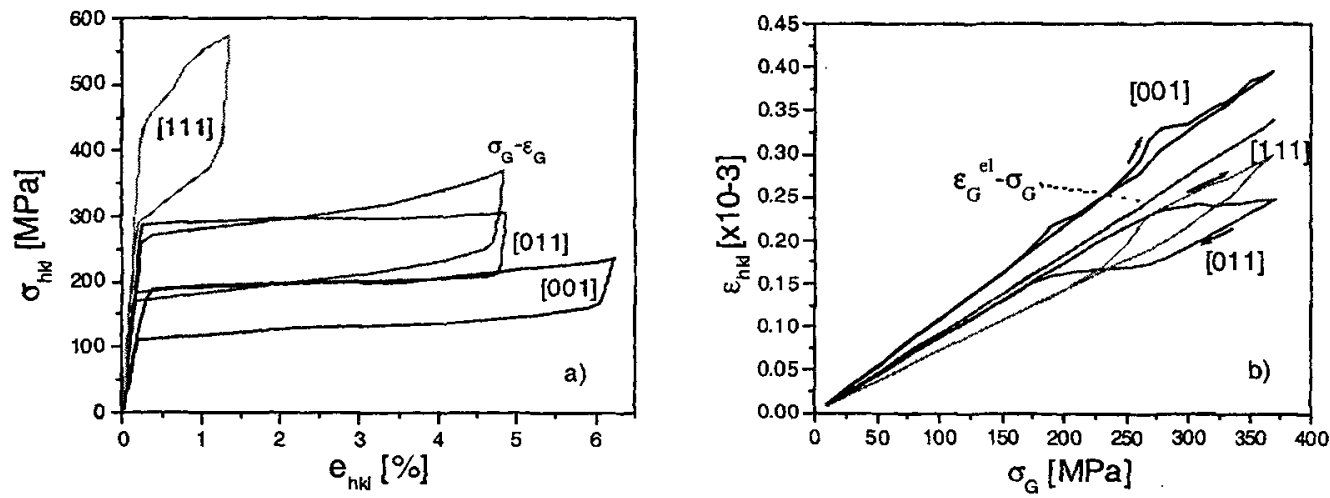

Figure 7: Simulation of the SIMT in CuAIZnMn polycrystal loaded in tension at $T>A_{f}: \sigma_{G}, \varepsilon_{G}$, stand for global macroscopic stress and strain. $\sigma_{h k t}, e_{h k l}$ are corresponding component values (selconsistent average values of stress and strain over the set of domains oriented with $h k l$-vectors parallel to the load axis direction) for [001],[011], and [111] oriented families of domains. $\varepsilon_{G}{ }^{\text {el }}$ is global elastic strain of the aggregate. 
stresses preceding the onset of the large scale SIMT $\left(\sigma_{G^{\sim}} 320 \mathrm{MPa}\right)$ in the CuAlZnMn polycrystal. The onset of the large scale transformation in the polycrystal $\left(\sigma_{G}>320 \mathrm{MPa}\right)$ is clearly evidenced by the integral intensities of most of the $h \mathrm{kl}$ austenite peaks starting to fall with the increasing stress, by the increased widening of the austenite peak profiles and by the appearance of the new martensite peaks in the spectra. The stresses corresponding to the stages I-III in the real polycrystal are slightly higher than predicted by the model. The decrease of the integral intensity $\left(I_{p, h k l} / I_{0, h k l}=\sim 0.6\right.$, Table 1$)$ similar for all $h k l$-reflections suggests that progress of the SIMT in the stage III is similar in most of the individual $h k l$ families of grains except the near [111] grains. This behavior is also supported by the modelling (Fig.7c,d). It means that no significant transformation induced texture but large intergranular stresses shall be expected in Cu-based SMA polycrystals transforming pseudoelastically in tension.

Let us point out finally that the above analysis of the load partition and "hardening" response of SMA polycrystal applies for the $\beta_{l} \rightarrow \beta_{l}^{\prime}$ transformation in Cu-based SMA polycrystals in tension, but need not apply at all for the compression or for other alloys as NiTi or NiAl [11]. This is because the transformation anisotropy is not a material characteristics of the austenite but depends on the type of the structural transition involved. The pseudoelastic response of the SMA polycrystal is thus predicted to be dependent, through the role of the elastic and transformation anisotropy in the load partition mechanism, on the applied stress state and on the grain orientation distribution - i.e. the stress-strain response will be also significantly affected by the sense of load and polycrystal texture.

\section{CONCLUSIONS}

In situ neutron diffraction study and modelling of the stress induced martensitic transformation in $\mathrm{CuAIZnMn} \mathrm{SMA} \mathrm{polycrystal} \mathrm{in} \mathrm{tension} \mathrm{suggests} \mathrm{that} \mathrm{stress} \mathrm{driven} \mathrm{transformability} \mathrm{of} \mathrm{the} \mathrm{polycrystalline}$ $\mathrm{Cu}$-based SMAs is controlled by mutual relationship and strengths of elastic anisotropy of the austenite phase and by the transformation anisotropy of the $\beta_{l} \rightarrow \beta_{l}^{\prime}$ martensitic transformation. The mechanism of the load partition among polycrystal grains in pseudoelasticity has been discussed and employed to clarify the origin of the pseudoelastic "hardening" of the CuAlZnMn polycrystal in the tensile load test.

Experimentally evaluated residual stresses in the austenite left behind from the first tensile cycle are claimed to assist the stress induced transformation in the subsequent tensile cyclic loads and held thus responsible for the significant increase in the pseudoleastic "hardening" slope between the first and second tensile load cycles.

\section{Acknowledgements}

Support of the Czech Ministry of Education (contract No. ME 186 (1999)), Grant Agency of the Czech Republic (contract 202/97/K038), and Grant Agency of the Academy of Sciences of the Czech Republic (contract No. A1010909) are greatly acknowledged.

\section{References}

1. K. Otsuka and C.M.Wayman, in Shape Memory Alloys, edited by K. Otsuka, C.M. Wayman, (Cambridge University Press, 1998), p.27.

2. V. Novák, P. Šittner, D. Vokoun and N. Zárubová, Mat. Sci. Eng. A, 273-275, 370 (1999).

3. K. Bhattacharya and R.V.Kohn, Acta mater. 44, 529 (1996).

4. M.R.Daymond,M.A.M.Bourke,R.B.VonDreele,B.Clausen,T.Lorentzen, J.Appl.Phys, 82, 1554 (1997).

5. A.N.Ezeilo and G.A.Webster, Textures and Microtextures, 33,151 (1999).

6. J.W.Hutchinson, Proc. R.Soc A319, 247 (1970).

7. P.A.Turner, N.Christodoulou and C.N.Tome, Int. J. Plasticity, 11, 251 (1995).

8. M.Daymond, C.N.Tome and M.A.M. Bourke, Acta Materialia,48, 553 (2000).

9. R.Vaydanathan,M.A.M.Bourke and D.C.Dunand, Acta Materialia, 47, 3353 (1999).

10. D. Neov, P. Šittner, P. Lukáš, V. Novák, P.Strunz, M. Vrána and P. Mikula, in Proceedings of $5^{t h}$ European Conference on Residual Stresses, Delft-Noordwijkerhout, 1999,

11. P Šittner and V Novák, International Journal of Plasticity, 16, (2000). 